J. Austral. Math. Soc. 19 (Series B), (1976), 469-477.

\title{
A DAM WITH GENERAL RELEASE RULE
}

\author{
GEOFFREY YEO
}

(Received 6 August 1976)

\begin{abstract}
A dam is considered with independently and identically distributed inputs occurring in a renewal process, and in particular a Poisson process, with a general release rate $r(\cdot)$ depending on the content. This is related to a $G I / G / 1$ queue with service times dependent on the waiting time. Some results are obtained for the limiting content distribution when it exists; these are more complete for some special release rates, such as $r(x)=\mu x^{\circ}$ and $r(x)=a+\mu x$, and particular input size distributıons.
\end{abstract}

\section{Introduction}

The waiting time $X(t)$ at time $t$ in a single server queue $G I / G / 1$, or the content of an equivalent dam, has been extensively studied [12]; there are more complete results for a Poisson arrival process $(M / G / 1)$. More general release rules than unit rate per unit time have also been considered [4], [7], [10], [11], [14], [15]; we consider an instantaneous release rate $r(X(t))$, which is a function of the content $X(t)$, at time $t$, such that $r(0-)=0$, and $r(x)$ is continuous and positive on $(0, \infty)$. For illustrative and numerical examples we shall consider the two special cases (i) $r(x)=\mu x^{\alpha}, 0<\mu<\infty, 0 \leqq \alpha<\infty$, so that the instantaneous release rate is proportional to the $\alpha$ th power of the content, and (ii) $r(x)=a+\mu x, 0 \leqq a<\infty, 0<\mu<\infty$ [10]. Case (i) with $\alpha=0$ and $\mu=1$ is $G I / G / 1$, and with $\alpha=1$ there is an exponential decay [8], [9], [14]; a variety of other values, such as $\alpha=1 / 2$ for a parallel sided sink, might be appropriate in particular circumstances. If (ii) $r(x)=1+\mu x$, then the second factor gives a way of providing faster service for large waiting times or content, and it also guarantees ergodicity.

We consider a stochastic process $X(t), 0 \leqq t<\infty$, called the content of a dam of capacity $K \leqq \infty$, defined on [0,K). Inputs, at $t_{1}<t_{2}<\cdots\left(t_{1}>0=t_{0}\right)$ occur in a renewal process with $\tau_{i}=t_{i+1}-t_{i}, i=0,1,2, \cdots$ being indepen- 
dently and identically distributed (i.i.d.) random variables with $P\left(\tau_{\imath} \leqq x\right)=$ $A(x), 0 \leqq x<\infty, E\left(\tau_{\imath}\right)=1 / \lambda, 0<\lambda<\infty$, and with Laplace transform (LT) $a^{*}(\theta)$. The inputs $S_{n}, n=1,2, \cdots$, are i.i.d. random variables with $P\left(S_{n} \leqq\right.$ $x)=G(x), 0 \leqq x<\infty, G(0)=0, E\left(S_{n}\right)=\beta<\infty$, and LT $g^{*}(\theta)$. When $A(x)=$ $1-\exp (-\lambda x)$, we have a compound Poisson input process.

The content $X(t)$ of a dam with infinite capacity and unit release rate is equivalent to the virtual waiting time in a single server queue. For a generai release rate this is no longer the case; the time a customer would wait for service depends on his own service time and possible subsequent arrivals. However, we may take $X(t)$ as a workload process and it is similar to the potential waiting process defined by Rubinovitch [13]. Let

$$
D(x)=\int_{y=0}^{x} \frac{1}{r(y)} d y \quad 0<x<\infty
$$

whenever the right-hand side is finite. Put $W(t)=D(X(t)), 0 \leqq t<\infty$. Then $W(t)$ is a process with slope- 1 except when inputs or overflow occur or when $W(t)=0 ; W(t) \geqq 0$ for all $t \geqq 0$ and $W(t)=0$ if and only if $X(t)=0$ [2]. The process may be interpreted as the virtual waiting time in a modified $G I / G / 1$ queueing system with service times depending on waiting times; it is thus an example of the important class of state dependent queueing systems.

We let $X\left(t_{n}-\right)=X_{n}, W\left(t_{n}-\right)=W_{n}$, and let $S_{n}^{*}\left(W_{n}\right)$ be the size of the $n$th input in the transformed process, i.e., "the service time of the $n$th customer" in the sense that it would take time $W_{n}+S_{n}^{*}\left(W_{n}\right)$ before the server became idle if no arrivals occurred in $\left(t_{n}, t_{n}+W_{n}+S_{n}^{*}\left(W_{n}\right)\right)$. As $W_{n}=D\left(X_{n}\right)$ and $W_{n}+S_{n}^{*}\left(W_{n}\right)=D\left(X_{n}+S_{n}\right)$ we find

$$
\begin{array}{ll}
S_{n}^{*}\left(W_{n}\right)=D\left(S_{n}+D^{-1}\left(W_{n}\right)\right)-W_{n} & \\
P\left\{S_{n}^{*}\left(W_{n}\right) \leqq x \mid W_{n}=w\right\}=G\left(D^{-1}(x+w)-D^{-1}(w)\right), & 0<x<\infty, \\
& 0 \leqq w<\infty,
\end{array}
$$

where $D^{-1}$ is the (unique) inverse function of $D(x)$, such that $D^{-1}(D(x))=x$; $S_{n}^{*}\left(W_{n}\right)$ is an increasing function of $D(x)$ and a decreasing function of $W_{n}$. For $K=\infty$ we have

$$
X_{n+1}=D^{-1}\left[\left\{D\left(X_{n}+S_{n}\right)-\tau_{n}\right\}^{+}\right]
$$

and

$$
W_{n+1}=\left\{W_{n}+S_{n}^{*}\left(W_{n}\right)-\tau_{n}\right\}^{+},
$$

which is Lindley's [12] form. Any results obtained for the original $X(\cdot)$ process may be interpreted in terms of the transformed $W(\cdot)$ process. 
In the special case (i) $r(x)=\mu x^{\alpha}(0 \leqq \alpha<1)$ and (ii) $r(x)=a+\mu x$, we have respectively

$$
\begin{aligned}
& D(x)=\frac{x^{1-\alpha}}{\mu(1-\alpha)}, D^{-1}(x)=\{\mu(1-\alpha) x\}^{1 /(1-\alpha)} \\
& S_{n}^{*}\left(W_{n}\right)=\left\{W_{n}^{1 /(1-\alpha)}+S_{n}(\mu(1-\alpha))^{\alpha-1}\right\}^{1-\alpha}-W_{n}, \\
& D(x)=\frac{1}{\mu} \ln \left(1+\frac{\mu x}{a}\right), D^{-1}(x)=\frac{a}{\mu}\left(e^{\mu x}-1\right) \\
& S_{n}^{*}\left(W_{n}\right)=\frac{1}{\mu} \ln \left\{1+\frac{\mu S_{n}}{a} e^{-\mu W_{n}}\right\} .
\end{aligned}
$$

If the integral in (1.1) is infinite, e.g., $r(x)=\mu x^{\alpha}, \alpha \geqq 1$, then the dam can not empty in finite time from any positive value of the content. For any $\varepsilon>0$ we put

$$
D_{f}(x)=\int_{y=}^{x} \frac{1}{r(y)} d y \quad 0<x<\infty
$$

with $D(x)=\lim _{\varepsilon \downarrow 0} D_{\varepsilon}(x)$ whenever the limit exists and is finite; in this case $D_{\varepsilon}(x)=D(x)-D(\varepsilon)$. For $x<\varepsilon$ we have $D_{f}(x)<0$, but $D_{f}(x)$ is still monotone in $x$. If we put $W_{\varepsilon}(t)=D_{\varepsilon}(X(t))$, then $W_{\varepsilon}(t)$ may be negative. However, at all relevant points in the argument below (also in [15]) $D_{\varepsilon}(x)$ actually occurs as a difference $D_{\varepsilon}(x)-D_{\varepsilon}(y)(x>0, y>0)$, which eliminates the dependence on $\varepsilon$. Consequently results can be justified for the more general case, although we shall argue only for the case $D(x)<x$ and shall leave the generalization to the reader.

We wish to study the distribution function (d.f.) $F\left(x, t ; x_{0}\right)=P\{X(t) \leqq$ $\left.x \mid X(0)=x_{0}\right\}$ and $H_{n}\left(x ; x_{0}\right)=P\left\{X_{n} \leqq x \mid X(0)=x_{0}\right\}$ of the content at time $t$ and before the $n$th input respectively, and the corresponding limiting d.f.'s $F(x)=\lim _{t \rightarrow \infty} F\left(x, t ; x_{0}\right)=P(X \leqq x)$ and $H(x)=\lim _{n \rightarrow \infty} H_{n}\left(x ; x_{0}\right)$, whenever they exist. By renewal theoretic arguments it follows [7] that $F(x)$ and $H(x)$ form proper d.f.'s whenever $K<\infty$ or $\lim _{x \rightarrow \infty} r(x)>\lambda \beta$. Further from (1.3) we have

$$
\begin{gathered}
H_{n+1}\left(x ; x_{0}\right)=\int_{y=0}^{\infty} d H_{n}\left(y ; x_{0}\right) P(y, x)=-\int_{y=0}^{\infty} H_{n}\left(y ; x_{0}\right) d_{y} P(y, x) \\
H(x)=-\int_{y=0}^{\infty} H(y) d_{y} P(y, x)
\end{gathered}
$$

where

$$
\begin{aligned}
P(y, x) & =P\left\{X_{n+1} \leqq x \mid X_{n}=y\right\} \\
& =\int_{w=0}^{\infty}\{1-A(D(y+w)-D(w))\} d G(w) .
\end{aligned}
$$


For $G I / G / 1$ we have $P(y, x)=P\left\{S_{n}-\tau_{n} \leqq x-y\right\}$ ([12]), p. 49), and the Wiener-Hopf equation (1.6) can be solved by known methods; in general (1.6) presents a much more complex problem.

Except for the special case $r(x)=\mu x$ we consider an exponential inter-input distribution; the input process is then compound Poisson (class 1 in [2]). Some general results are given in the next section, with more explicit and numerical examples for some special input size distributions in Sections 3 and 4 .

\section{Compound Poisson input process}

In the case of an exponentially distributed time between inputs we can formally obtain an integro-differential equation [7] for $F(x, t)$, which for $K=\infty$ has been solved in some special cases, such as for $r(x)=1(M / G / 1)[12]$ and in terms of LT's for $r(x)=\mu x$ [8], [9]. For the limiting content distribution we have

$$
r(x) F^{\prime}(x)=\lambda F(x)-\lambda \int_{y=0}^{x} F(x-y) d G(y) \quad 0<x<K,
$$

provided $K<\infty$ or $\lim _{x \rightarrow \infty} r(x)>\lambda \beta$. For the transformed process $W=D(X)$ with $L(w)=P\{W \leqq w\}$ we have

$$
L^{\prime}(w)=\lambda L(w)-\lambda \int_{u=0}^{w} L(u) d G\left(D^{-1}(w)-D^{-1}(u)\right), \quad 0<w<D(K) .
$$

For the remainder of this section we suppose $K=\infty$. We define $\psi(\theta)=$ $\int_{0}^{\infty} \exp (-\theta x) d F(x)(0 \leqq \theta<\infty)$ as the LT of the limiting content; from (2.1)

$$
\begin{gathered}
\int_{x=0-}^{\infty} r(x) e^{-\theta x} d F(x)=r(0) F(0)+\psi(\theta) \zeta(\theta) \\
\zeta(\theta)=\rho\left\{1-g^{*}(\theta)\right\} / \theta .
\end{gathered}
$$

The LT $\psi(\theta)$ is known in the special cases $r(x)=1$ [12] and $r(x)=\mu x$ [8]. In the combined case $r(x)=\alpha+\mu x$ (2.3) gives

$$
\psi^{\prime}(\theta)+(\zeta(\theta)-\gamma) \psi(\theta)=-\gamma F(0)
$$

where $\gamma=a / \mu, \rho=\lambda / \mu$. Using $\psi(0)=1$ we obtain

$$
\begin{aligned}
& \psi(\theta)=e^{-\jmath(\theta)}\left\{1-\gamma F(0) \int_{y=0}^{\theta} e^{J(y)} d y\right\} \\
& J(\theta)=-\gamma \theta+\int_{y=0}^{\theta} \zeta(y) d y .
\end{aligned}
$$


As a consequence of $E(S)=\beta<\infty$ we find $-J(\theta) \rightarrow+\infty$ as $\theta \rightarrow \infty$, so that

$$
F(0)=\left\{\gamma \int_{y=0}^{\infty} e^{J(y)} d y\right\}^{-1}
$$

The procedure can be used for more general input processes [3]. When $g^{*}(\theta)=\nu /(\nu+\theta)$ this gives (3.6) at $x=0$, while for $g^{*}(\theta)=\exp (-\theta \beta) F(0)$ can be evaluated numerically. Moments may be obtained by

$$
\begin{aligned}
& E(X)=\rho \beta-\gamma(1-F(0)) \\
& V(X)=\frac{\rho E\left(S^{2}\right)}{2}+(\rho \beta-\gamma) E(X) .
\end{aligned}
$$

From (2.1) it follows that

$$
E(r(X))=\lambda \beta+r(0+) F(0),
$$

which gives for $r(x)=\mu x^{\alpha}(\alpha>0)$ that

$$
\begin{aligned}
& E\left(X^{\alpha}\right)=\rho \beta \\
& E\left(X^{\alpha+1}\right)=\rho \beta E(X)+\frac{\rho}{2} E\left(S^{2}\right),
\end{aligned}
$$

etc., which involves finding the moments of integer order. For $\alpha=0$ (and $\rho \beta<1)$ and $\alpha=1$ all moments can be obtained in this way by recurrence. Further if $r(x)=\mu x$ all moments can be found for inputs occurring in a renewal process; if $X^{*}$ is the content just before an input occurs, then.(see [6])

$$
\begin{aligned}
& E\left(X^{* r}\right)=\frac{a^{*}(r \mu)}{1-a^{*}(r \mu)} \sum_{j=0}^{r-1}\left(\begin{array}{l}
r \\
j
\end{array}\right) E\left(X^{* j}\right) E\left(S^{r-1}\right) \\
& E\left(X^{r}\right)=\frac{\rho\left\{1-a^{*}(r \mu)\right\}}{r} \sum_{j=0}^{r}\left(\begin{array}{l}
r \\
j
\end{array}\right) E\left(\dot{X}^{* \prime}\right) E\left(S^{\prime-i}\right) .
\end{aligned}
$$

In the case of $r(x)=\mu x^{\alpha}(0<\alpha<1)$ we can use a fractional LT ([5], Section 4.7) to obtain

$$
\lim _{c \rightarrow \infty} \int_{t=\theta}^{c} \frac{(1-\theta)^{-\alpha}}{\Gamma(1-\alpha)} \psi^{\prime}(t) d t=-\zeta(\theta) \psi(\theta) .
$$

By differentiation we obtain formally

$$
\rho \beta E(X)+\frac{\rho}{2} E\left(S^{2}\right)=\int_{t=0}^{\infty} \frac{t^{-\alpha}}{\Gamma(1-\alpha)} \psi^{\prime \prime}(t) d t,
$$

which gives known results as $\alpha \rightarrow 0$ or 1 . 


\section{Exponentially distributed inputs}

We assume that the size of an input has a negative exponential ditribution with mean $\beta=1 / \nu, 0<\nu<\infty$, so $G(x)=1-\exp (-\nu x), 0 \leqq x<$ $\infty$. From (2.1)

$$
r(x) F^{\prime}(x)=\lambda F(x)-\nu \lambda e^{-\nu x} \int_{y=0}^{x} F(y) e^{\nu y} d y, \quad 0<x<K,
$$

and hence by differentiation

Thus

$$
F^{\prime \prime}(x)+\left(\nu+\frac{r^{\prime}(x)}{r(x)}-\frac{\lambda}{r(x)}\right) F^{\prime}(x)=0, \quad 0<x<K .
$$

$$
\begin{aligned}
& F^{\prime}(x)=\operatorname{cr}(x)^{-1} e^{O(x)} \\
& Q(x)=-\nu x+\lambda D(x), \quad 0<x<K
\end{aligned}
$$

and using $F(K)=1$ and (2.6) we find

$$
F(x)=\frac{e^{O(x)}+\nu \int_{y=0}^{x} e^{O(y)} d y}{e^{O(K)}+\nu \int_{y=0}^{K} e^{O(y)} d y} \quad 0 \leqq x \leqq K,
$$

which has been obtained by McNeil [10], p. 253, using a limiting result of another problem. From (3.3) or (2.3) the equivalent result for $L(z)$ can easily be found.

More explicit results can be found for special cases of the release rate $r(x)$; for convenience we let $K=\infty$. When $r(x)=1$ (and $\lambda / \nu<1$ ) $F(x)=$ $1-(\lambda / \nu) \exp (\nu-\lambda) x$, and when $r(x)=\mu x$ (3.3) is (truncated) gamma [8]. If $r(x)=\mu \vee x$, i.e., $\alpha=1 / 2$, and if $\phi(x)$ and $\Phi(x)$ are the density function and distribution function of a standard normal random variable, and $\gamma=$ $\rho \sqrt{ }(2 / \nu)$, it follows that $F(0)=\phi(\gamma) /(\phi(\gamma)+\gamma \Phi(\gamma))$, and $Y=$ $\mathcal{V}(2 \nu X)-\gamma=\sqrt{ } \nu W-\gamma$ is a truncated standard normal random variable on $(-\gamma, \infty)$ with density function $\gamma F(0) \phi(y) / \phi(\gamma)$ with a jump of size $F(0)$ at $-\gamma$. Further $E(\vee X)=\rho \beta(2.6)$ and

$$
\begin{gathered}
E(X)=\frac{\gamma F(0)}{2 \nu \phi(\gamma)}\left\{2 \gamma \phi(\gamma)+\gamma^{2} \Phi(\gamma)+1-\frac{1}{2} Q\left(\gamma^{2} ; 3\right)\right\} \\
E\left(X^{2}\right)=\frac{\gamma F(0)}{4 \nu^{2} \phi(\gamma)}\left\{1+6 \gamma^{2}+\left(8 \gamma+8 \gamma^{3}\right) \phi(\gamma)+\gamma^{4} \Phi(\gamma)-3 \gamma^{2} Q\left(\gamma^{2} ; 3\right)\right. \\
\left.-\frac{1}{2} Q\left(\gamma^{2} ; 5\right)\right\},
\end{gathered}
$$

where $Q\left(x^{2} ; k\right)$ is.the tail of the gamma function with index $k / 2$ ([1], p. 978). 
If $r(x)=\mu x^{2}$, i.e., $\alpha=2$, then $F(0+)=0$, and with $\sigma=\rho \nu=\lambda \nu / \mu$ we obtain

$$
F^{\prime}(x)=\frac{V \rho e^{-\nu x-\rho / x}}{2 x^{2} \vee \nu K_{1}(2 \sqrt{\rho \nu})} \quad 0<x<\infty,
$$

where $K_{,}(x)$ is the modified Bessel function of the second kind and of order $j$ ([1], p. 417). The constant $c^{-1}$ in (3.2) is given by the solution of $a^{\prime \prime}(\sigma)=$ $a(\sigma) / \sigma$, where

$$
a(\sigma)=\int_{y=0}^{\infty} e^{-(y+\sigma / y)} d y=2 \vee \sigma K_{1}(2 \vee \sigma) .
$$

In this case $E\left(X^{2}\right)=\rho \beta(2.6)$ and

$$
\begin{aligned}
E(X) & =c \int_{x=0}^{\infty} \frac{1}{x} e^{-(\nu x+\rho / x)} d x=\frac{-\rho a^{\prime}(\sigma)}{a(\sigma)} \\
& =\sqrt{\frac{\rho}{\nu}} \frac{K_{0}(2 \vee \sigma)}{K_{1}(2 \sqrt{ }(2)} .
\end{aligned}
$$

TABLE 1

The mean $E(X)$, standard deviation $\vee V(X)$ and probability $F(0+)$ of emptiness for exponentially distributed $(\nu)$ inputs with $r(x)=\mu x^{\alpha}, \alpha=0, \frac{1}{2}, 1,2, \rho=\lambda / \mu=0.5$, $1,2,4$, and $r(x)=1+x \rho$.

\begin{tabular}{cccccl}
$\rho$ & $\nu$ & $\alpha$ & $E(X)$ & $V V(X)$ & $F(0+)$ \\
\hline 0.5 & 1 & 0 & 1 & 1.732 & 0.500 \\
& & $\frac{1}{2}$ & 0.567 & 0.960 & 0.367 \\
& & 1 & 0.500 & 0.707 & 0 \\
& & 2 & 0.537 & 0.452 & 0 \\
1 & 1 & $x / 2$ & 0.302 & 0.639 & 0.604 \\
& & 1 & 1.449 & 1.588 & 0.103 \\
& & 1 & 1 & 1 & 0 \\
2 & 1 & 0.814 & 0.580 & 0 \\
& & $1+x$ & 0.500 & 0.866 & 0.500 \\
& & 1 & 4.499 & 2.919 & 0.0028 \\
4 & & 2 & 1.212 & 0.728 & 0 \\
& 1 & $1+2 x$ & 0.800 & 1.166 & 0.400 \\
& & $\frac{1}{2}$ & 16.500 & 5.701 & 0.000 \\
& & 1 & 4 & 2 & 0 \\
1 & & 2 & 1.788 & 0.896 & 0 \\
& & $1+4 x$ & 1.243 & 1.567 & 0.311 \\
& 2 & 0 & 0.500 & 1.225 & 0.500 \\
& & $\frac{1}{2}$ & 0.444 & 0.610 & 0.223 \\
& & 1 & 0.500 & 0.500 & 0 \\
& & 2 & 0.606 & 0.364 & 0 \\
& & $1+x$ & 0.167 & 0.373 & 0.667
\end{tabular}


When $r(x)=a+\mu x$ we find

$$
F(x)=\frac{\Gamma\left(\frac{\nu a}{\mu}+\nu x ; \rho+1\right)-\Gamma\left(\frac{\nu a}{\mu} ; \rho+1\right)+\left(1+\frac{\mu x}{a}\right)^{\rho} e^{-\nu x} \gamma\left(\frac{\nu a}{\mu} ; \rho+1\right)}{1-\Gamma\left(\frac{\nu a}{\mu} ; \rho+1\right)}
$$

where $\gamma(x ; \rho)$ and $\Gamma(x ; \rho)$ are respectively the density function and the distribution function of a gamma distribution with index $\rho(\Gamma(x ; \rho)=$ $1-Q(2 x ; 2 \rho)([1]$, p. 978)).

For $r(x)=\mu x^{\alpha}, \alpha=0, \frac{1}{2}, 1,2$, and $r(x)=1+\rho x$ and a selection of values of $\rho$ and $\nu$ the probability of emptiness $F(0+)$, the mean $E(X)$ and the standard deviation $\vee V(X)$ of the content are shown in Table 1.

\section{Bounded inputs}

We consider inputs which have zero mass on $[0, \eta), 0<\eta<\infty$, with mean $\beta$; many input distributions would satisfy such a mild restriction. Further this would give an approximation when counting only inputs of magnitude at least $\eta$ occurring in a stable input process as defined in [2]. In this case (2.2) can be solved iteratively over $[0, \eta),[\eta, 2 \eta), \cdots$ to obtain

$$
F(x)=\frac{e^{\lambda D(x)} \sum_{j=0}^{|x / \eta|}(-1)^{\prime} \xi_{j}(x)}{e^{\lambda D(K)} \sum_{j=0}^{|K / \eta|}(-1)^{\prime} \xi_{j}(K)} \quad 0 \leqq x \leqq K
$$

where $\xi_{0}(x)=1,0 \leqq x<\infty$ and

$$
\xi_{l}(x)=\lambda \int_{w=/ \eta}^{x} \frac{e^{-\lambda D(w)}}{r(w)} d w \int_{y=(-1) \eta}^{w-\eta} e^{\lambda D(y)} \xi_{l-1}(y) d G(w-y) \quad j \eta \leqq x<\infty .
$$

If the inputs are constant, i.e., $G(x)=1$ for $x \geqq \eta=\beta$, then

$$
\xi_{1}(x)=\lambda \int_{y=j \beta}^{x} \frac{e^{-\lambda D(y)+\lambda D(y-\beta)}}{r(y)} \xi_{j-1}(y-\beta) d y \quad j \beta \leqq x<\infty .
$$

For $r(x)=\mu x^{\alpha}, \alpha=0,0.25,0.50,0.75,1,1.50,2, \beta=1, \rho=0.50,1,2,4$, and $x$ an integer $\leqq 7(4.2)$ has been evaluated [14], [15].

If the interinput is Erlang with index $k(=2,3, \cdots)$, then the iterative procedure used above carries over.

\section{Acknowledgements}

I am grateful to P. J. Brockwell, B. D. Craven and N. M. H. Smith for helpful comments and discussion. 


\section{References}

[1] M. Abramowitz and I. Stegun, Handbook of Mathematical Functions (National Bureau of Standards, 1964).

[2] P. J. Brockwell and K. L. Chung, 'Emptiness times of a dam with stable input and general release function', J. Appl. Prob. 12 (1975), 212-217.

[3] P. J. Brockwell, 'Stationary distributions for dams with additive input and general release rule' (submitted).

[4] E. Cinlar and M. Pinsky, 'On dams with additive inputs and a general release rule', J. Appl. Prob. 9 (1972), 422-429.

[5] R. Courant. Differential and Integral Calculus, Vol. 2, Blackie, 1936.

[6] J. L. Gastwirth and S. S. Wolff, 'On the relation between a particular stochastic process and its two embedded Markov processes', Sankhya A 28 (1966), 151-156.

[7] D. P. Gaver and R. G. Miller, 'Limiting distributions for some storage problems', in: K. J. Arrow, S. Karlin and H. Scarf, ed. Studies in Probability and Management Science Stanford U. P., 1962.

[8] S. Karlin, A First Course in Stochastic Processes, Academic Press, 1966.

[9] J. Keilson and N. D. Mermin, 'The second-order distribution of integrated shot noise'. IRE Tran. on Information Theory, IT-5 (1959), 75-77.

[10] D. R. McNeil, 'A simple model for a dam in continuous time with Markovian inputs', $Z$. Wahrscheinlichkettstheorie verw. Geb. 21 (1972), 241-254.

[11] P. A. P. Moran, 'A theory of dams with continuous input and a general release rule', J. Appl. Prob. 6 (1969) 88-93.

[12] N. U. Prabhu, Queues and Inventories, (Wiley, 1965).

[13] M. Rubinovitch, 'Queues with random service output', Adv. Appl. Prob. 6 (1974), 207-208.

[14] G. F. Yeo, 'A finite dam with exponential release', J. Appl. Prob. 11 (1974), 122-133.

[15] G. F. Yeo, 'A finite dam with variable release rule', J. Appl. Prob. 12 (1975), 205-211.

Department of Mathematics,

Odense University,

Odense,

Denmark. 